\title{
Photonics in nature: Yellowstone National Park in IR
}

Michael Vollmer, Joseph Shaw, Paul Nugent, Wilson Harris, Kendra Gillis, et al.

Michael Vollmer, Joseph A. Shaw, Paul W. Nugent, Wilson Harris, Kendra Gillis, William Weiss, Logan Carpenter, Amy Carpenter, Bryan Scherrer, "Photonics in nature: Yellowstone National Park in IR," Proc. SPIE 10452, 14th Conference on Education and Training in Optics and Photonics: ETOP 2017, 104521B (16 August 2017); doi: 10.1117/12.2266677

SPIE Event: 14th Conference on Education and Training in Optics and Photonics, ETOP 2017, 2017, Hangzhou, China 


\title{
Photonics in Nature: Yellowstone National Park in IR
}

\author{
Michael Vollmer ${ }^{\mathrm{a}}$, Joseph A. Shaw ${ }^{\mathrm{b}}$, Paul W. Nugent ${ }^{\mathrm{b}, \mathrm{c}}$, Wilson Harris ${ }^{\mathrm{b}}$, Kendra Gillis ${ }^{\mathrm{d}}$, William \\ Weiss ${ }^{\mathrm{d}}$, Logan Carpenter ${ }^{\mathrm{d}}$, Amy Carpenter ${ }^{\mathrm{e}}$, Bryan Scherrer ${ }^{\mathrm{b}}$ \\ ${ }^{a}$ University of Applied Sciences, Brandenburg, Magdeburgerstr. 50, 14470 Brandenburg, Germany \\ ${ }^{b}$ Electrical and Computer Engineering Department, Montana State University, Bozeman, Montana, USA 59717 \\ ${ }^{\circ}$ NWB Sensors, Inc., Bozeman Montana, USA 59715 \\ ${ }^{\mathrm{d}}$ Physics Dept., Brigham Young University, Rexburg, Idaho, USA 83460 \\ ${ }^{\mathrm{e}}$ Mathematics Dept., Brigham Young University, Rexburg, Idaho, USA 83460
}

\begin{abstract}
Infrared thermal imaging is a valuable tool not only in science but also in optics and photonics education and outreach activities. Observing natural optical phenomena in a different spectral region like the thermal infrared often offers new insights. The commonly used false color images not only allow extraction of useful information about thermal properties of objects, but they can also provide aesthetic sights and are thus an excellent tool for public outreach activities. Recently we have pursued this kind of study using IR imaging within Yellowstone National Park, complementing earlier work on thermal pool colors and spectroscopy. We will discuss and compare images of a variety of VIS and IR cameras of hot springs, geysers, mud pools and other natural phenomena recorded in the park during 2012 and 2016.
\end{abstract}

Keywords: Optics education, infrared imaging, Yellowstone National Park, Hot Springs, Geysers

\section{1) INTRODUCTION}

In the past decades, infrared thermal imaging has become an extremely valuable tool not only in science but also in optics and photonics education ([1,2] and refs. therein). Of course, IR cameras can also be used to study objects in nature such as clouds [3], the moon [4], mirages [5] and many other natural phenomena. As an example, IR imaging was combined with spectroscopy to reveal the relation of color and temperature in hot thermal pools in Yellowstone National Park [6,7]. These latter studies revealed that IR imaging may additionally serve as an excellent tool for outreach activities. We now have extended this work and gathered new IR imagery of Yellowstone National Park's thermal features [8]. Here we will present and discuss some respective examples which have educational use in optics and also value for science outreach activities. This kind of outreach makes use of the generally high interest found among a wide range of people regarding beautiful optical phenomena in nature [9].

Geothermal features can of course always be appreciated by visual observation and visible imagery. However, the colorful hot pools and the steam above them, or the huge water steam clouds observed during geyser eruptions, are only a small part of the underlying beauty of these natural phenomena. Even more information about the underlying physics can be gained using infrared imaging. Suddenly, one is able to also see that the colorful pools are extremely hot, some of them near boiling temperature. And the geysers emit not only steam, but tons of hot water as well. Similarly, additional information can be gathered by observing many other natural phenomena, and therefore the infrared eyes of IR cameras open up completely new perspectives to enhance our vision of nature beyond our usually perceived visual range. This new vision creates curiosity and attracts people. For example, at Yellowstone Park it took us nearly as long to answer questions from passers-by about what we were doing as did our actual recording of the phenomena with IR imaging. Presenting this imagery always attracts not only people's eyes, but also their minds, and they are usually interested enough to even listen and view simple physics interpretations. Besides, people want to know more about the technology, which is also becoming more well-known in other aspects of our daily life, such as, e.g., thermal insulation inspections of private homes. As a consequence, thermal imagery of natural phenomena, in this case, Yellowstone's National Park thermal features, is an excellent example of how modern technology can be used as an extremely attractive public outreach tool. 


\section{2) THERMAL CAMERAS USED IN THIS STUDY}

Many different infrared cameras are commercially available and they are rapidly becoming commonplace in modern society. They differ in wavelength range, pixel numbers, sensitivity and whether they are used qualitatively or quantitatively, i.e. whether they are radiometrically calibrated or not. In addition, many different software tools are available for analysis. The governing factor for all these properties is the price of the camera.

The present study was conducted with small and rather inexpensive thermal cameras that use uncooled Microbolometer detectors to record thermal radiation in the approximate wavelength range of 8-14 $\mu \mathrm{m}$. Off the shelf, these cameras come mostly without highly accurate radiometric calibration. However, we have developed methods of calibrating these cameras and stabilizing their output to be largely independent of variations in the camera temperature [10,11]. Such calibrations have been applied to the images shown here, which produce much more uniform and quantitatively useful images than would be obtained otherwise.

Another characteristic of these small thermal cameras is their low pixel count. The images shown here were recorded with a FLIR Photon 320 camera $(324 \times 256$ pixels), a FLIR photon 640 camera $(644 \times 512$ pixels), and a FLIR Tau2 336 camera $(324 \times 256$ pixels). To improve the spatial resolution in IR images, we stitched multiple frames together when imaging static scenes. Furthermore, each photo in the stitched Photon 320 images is an average of 10 frames to reduce noise. For the Tau336 camera, each image is an average of approximately 200 frames ( $5 \mathrm{~s}$ on each position at $40 \mathrm{fps}$ ).

\section{3) SELECTED THERMAL FEATURES IN YELLOWSTONE NATIONAL PARK}

Yellowstone National Park located in the US states of Montana, Idaho and Wyoming is not only the world's first national park, it is probably also the most well-known world-wide. Yellowstone's geothermal features are breath-taking and inspiring, as much art as science, in particular if observed with close-up infrared thermal imaging. Here we present a few examples which are particularly suitable for public outreach activities. Still we occasionally add some relevant physics background information, which may also be used in undergraduate teaching. Some corresponding IR video files are available at the OPN website [12].

\section{a) Geysers}

The most well-known feature in Yellowstone National Park is undoubtedly Old Faithful, which is probably also the world's most famous geyser. Geysers are more or less water filled cavities extending tens of meters below the surface of the earth. The boiling temperature within the water column varies with depth as it is pressure dependent. Being heated from below, the water first starts to boil at the bottom of the cavity. Vapor bubbles rise which accelerate further boiling and finally lead to giant eruptions, which in the case of Old Faithful can reach heights of up to 50 meters. Figure 1 depicts an example of an eruption recorded with a long wave IR camera and a typical visible image of such an eruption.
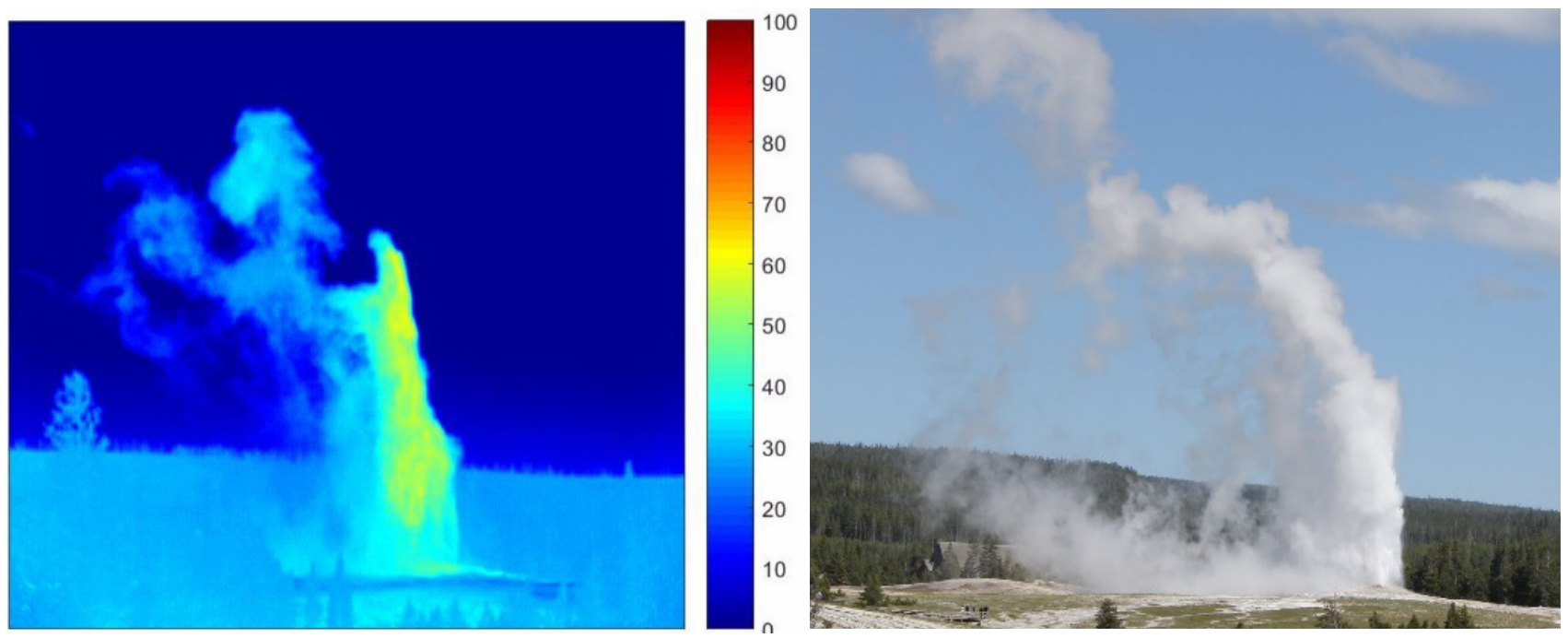

Fig. 1: Eruption of the Old Faithful geyser in IR and VIS. The IR image was recorded with a 644 x 512 pixel LWIR camera from a distance of about $280 \mathrm{~m}$. The VIS image shows a different but similar eruption. 


\section{b) Hot pools and springs}

Although geysers are maybe the most spectacular, a large percentage of Yellowstone's unique landscape is covered by smaller features that also offer fantastic views. Consider, for example, the IR and VIS images in Fig. 2, which show Silex Spring in the Lower Geyser Basin. This is an example of a rather quiet spring with dimensions of approximately 10 $\mathrm{m} \times 12 \mathrm{~m}$, a maximum depth near $8 \mathrm{~m}$, and with discharges up to $200 \mathrm{l} / \mathrm{min}$. Its maximum temperature is quoted to be around $193{ }^{\circ} \mathrm{F}$, i.e. about $89^{\circ} \mathrm{C}$. Being quiet, it may be easily observed from nearby. Here we recorded images from a boardwalk with a distance of around $8 \mathrm{~m}$ to $10 \mathrm{~m}$ to the center of the pool. The camera was on a tripod at a height of about $1.5 \mathrm{~m}$, which means that the angle of incidence of the camera's view with respect to the vertical direction was around $80^{\circ}$.

The IR image in Fig. 2 was recorded with a FLIR Photon 320 camera and a lens with $50^{\circ}$ full-angle field of view. It is a composite of five partially overlapping images, each containing $324 \times 256$ pixels, with the composite containing around $660 \times 310$ pixels. The typical jagged edges due to the image stitching process have been removed by cropping. This image illustrates that many thermal pools can reach very high temperatures; here the water temperature is observed to be close to $90^{\circ} \mathrm{C}$, which is not too far away from the boiling temperature of water, which at the elevation of Yellowstone Park is around $92{ }^{\circ} \mathrm{C}$.
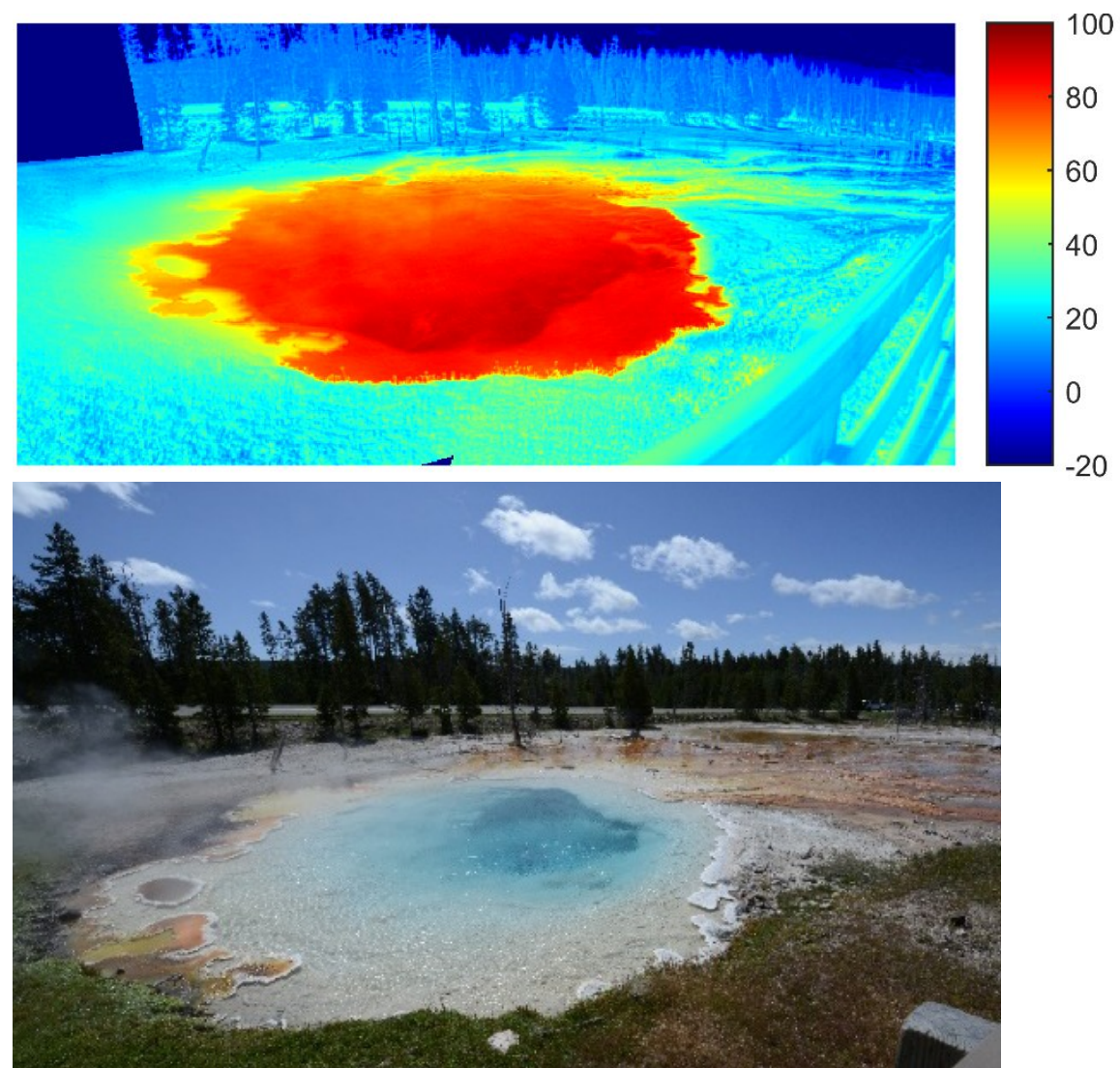

Fig. 2: IR and VIS image of Silex pool in the Lower Geyser basin (the colors indicate temperature).

The IR image of Fig. 2 is also interesting from the physics point of view and it may explain why the currently available IR camera smartphone accessories, or the simple IR thermometers that can be purchased or rented in Yellowstone Park to check the temperature of thermal features, sometimes give wrong - mostly too low - temperature readings.

An IR camera is usually calibrated with blackbody sources of well-defined temperature, which are observed at normal incidence. If an object of unknown temperature is observed at normal incidence, its temperature can be estimated if the material property of emissivity is properly accounted for. Most objects - and water belongs to this class - are 
characterized by an emissivity that varies fairly slowly with wavelength. Therefore, one would guess that once the proper emissivity value is used, an IR measurement would give the correct result. Unfortunately, however, emissivity depends on the angle of emission with respect to the surface normal. Its value can drastically decrease for large angles. For example, if water has an emissivity of approximately 0.98 at normal incidence (i.e., a flat pool surface viewed from the zenith), this value would decrease to about 0.6 at the $80^{\circ}$ incidence angle used to record Fig. 2 . Therefore, correct temperature readings must account for this lowered emissivity which also go along with colder background sky reflection contributions. Cameras do not perform this correction automatically, but this is rather a task for the user. In this case, the water has a normal-incidence emissivity of approximately 0.98 . If we would have observed the pool at $80^{\circ}$ angle with this emissivity value, the camera reading would have been only about $60{ }^{\circ} \mathrm{C}$, rather than the correct value close to $90^{\circ} \mathrm{C}$.

Finally, we note that a lower emissivity also corresponds to a higher reflectivity, so the IR emission from the reflected background must also be included in a careful measurement of the water temperature. When that reflected background is clear sky, the reflected component can remain much lower than the emitted component from hot water, even at relatively large viewing angles.

Figure 3 depicts Elephant Mound, better known as Orange Spring Mound in the Mammoth terraces area. The outflow temperature at the left hand side of the mound barely reaches $70{ }^{\circ} \mathrm{C}$ and allows orange colored cyanobacteria to grow, giving the color and the name for this mound. The IR image was recorded with a FLIR Tau2 336 camera with a $17^{\circ}$ field-of-view lens. It is a composite of overall 33 partially overlapping images, each containing $324 \times 256$ pixels, with the cropped composite containing about $1350 \times 480$ pixels. From a distance, one may already detect the outflow due to the visibly colorful bacterial mat; however, the IR image yields the additional information about its temperature.
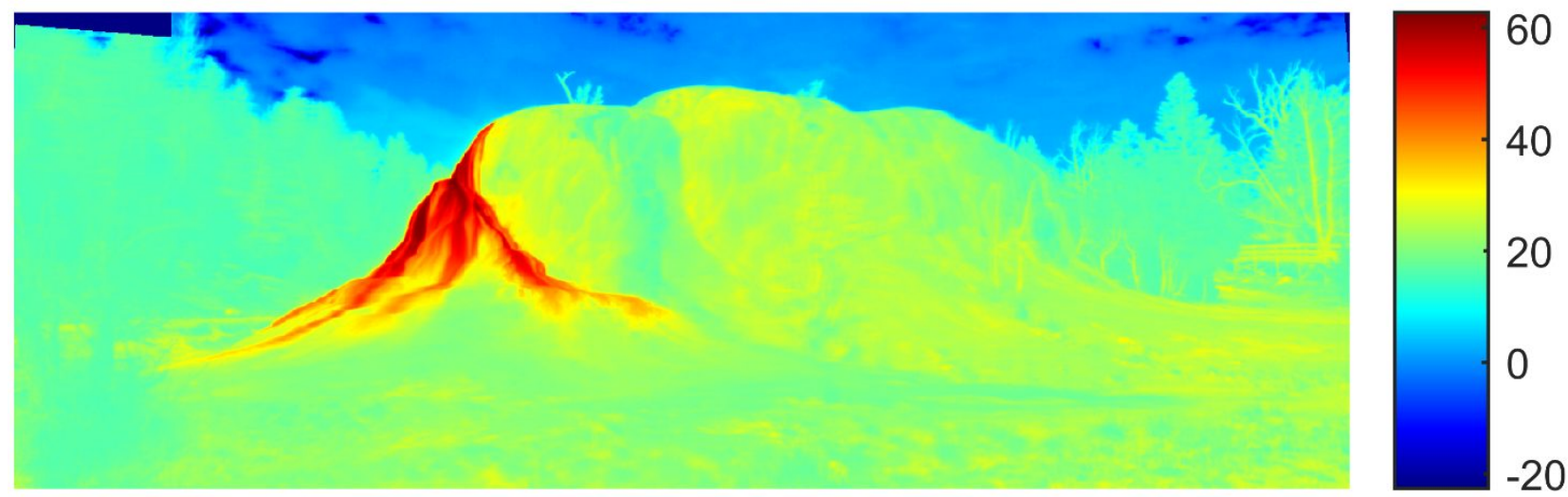

Fig. 3: Elephant Mound Spring in the Mammoth Terraces area.

\section{c) Hot inflow into cold rivers}

At the intersection of hot water runoffs from hot pools and geysers with cold rivers, thermal images allow us to visualize the physics principles of turbulence. For example, although it can be fascinating to watch the near-boiling water from Excelsior Geyser flow into the cold water of the Firehole River (approximately $16{ }^{\circ} \mathrm{C}$ in our images) near the parking lot of Grand Prismatic Spring, it becomes even more fascinating in the infrared. The thermal images in Fig. 4 show turbulent mixing processes that could easily as well depict hot lava outflows in Hawaii entering the sea.

The IR images were recorded with a FLIR Photon 640 camera with a $10^{\circ}$ field-of-view lens. Each image contains $644 \times$ 512 pixels. Due to the rapid changes of the scene, we did not produce any composite images. The visible photographs help the reader see how dramatically the same scene differs in the visible and IR. These images were recorded with viewing distances of $32 \mathrm{~m}$ from the opposite riverbank (left) and $8 \mathrm{~m}$ from the nearby walking bridge (right). 

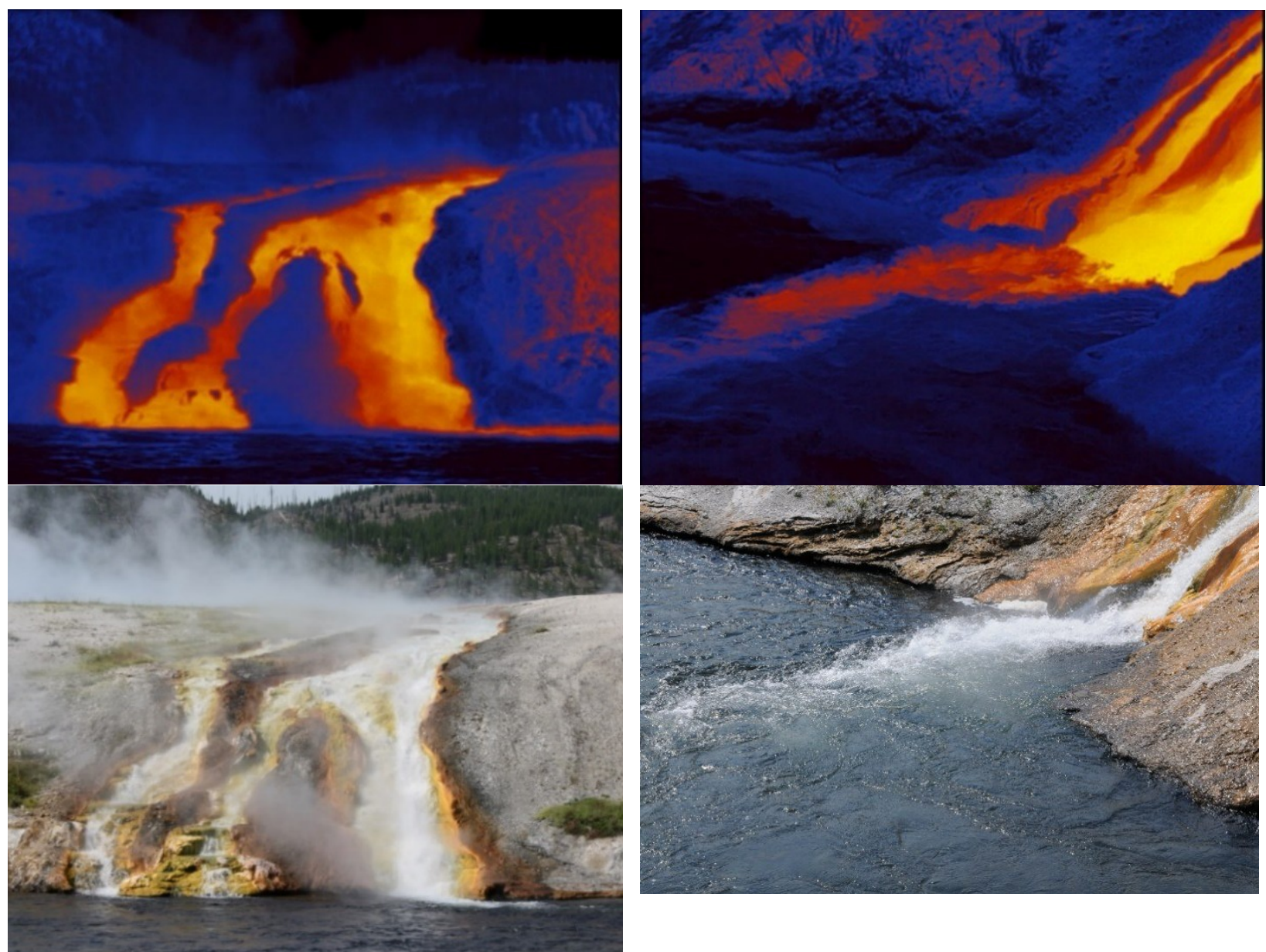

Fig. 4: IR (top) and VIS (bottom) images of hot water from the Excelsior Geyser outflow mixing with the cold Firehole River: viewed from the opposite bank (left) and from a nearby bridge (right).

\section{d) HOT and COLD: the Grand Canyon of the Yellowstone!}

Besides erupting geysers and hot water flows, Yellowstone National Park also offers other fascinating geological features such as the Grand Canyon (of the Yellowstone) and numerous water falls. Among these features, IR imaging can reveal surprising and unexpected hot and cold spots. For example, Fig. 5 shows an overview of the northern canyon walls observed from Artist Point on the southern rim. The visible image (bottom) depicts a colorful canyon wall with faint pastel colors of yellow and pink shades below a partly cloudy blue sky. The distance from the camera to the opposite canyon wall varies between around $450 \mathrm{~m}$ at the left to a maximum around $870 \mathrm{~m}$ at the upper right hand side. Deep within the canyon, a few parts of the Yellowstone River are visible. The infrared image of Fig. 5 (top) was recorded with a FLIR Tau2 $336 \mathrm{LWIR}$ camera with a $17^{\circ}$ field-of-view lens. As a matter of fact, it is a composite of overall 27 partially overlapping images, each containing $324 \times 256$ pixels, with the cropped composite containing about $1145 \times 690$ pixels. An individual IR pixel corresponds to about $40 \mathrm{~cm}$ at the left to a maximum around $80 \mathrm{~cm}$ at the upper-right-hand-side rocks.

The IR image in Fig. 5 shows some characteristic features. First, as already also seen in Figs. 1 to 3, the sky always appears dark, i.e. of low temperature, and clouds show a very good contrast with respect to the clear sky. This is characteristic of IR sky images with fairly dry air [1]. Second, looking closely, one can immediately detect the colder river. Third, there are two hot spots on the IR image. Unfortunately, only one of them, is within the field of view of the VIS images, as the VIS camera was displaced from the IR camera and the right edge hot spot is hidden behind vegetation in the VIS image. The other hot spot on the canyon wall, located a little bit left of the IR image center, is also 
in the field of view of the VIS camera image. However, even enlarging it to much higher spatial resolution, the VIS image does not show any specific feature which may be attributed to it. We interpret it as a thermal feature, either an outflow or some hot gas plume, heating the surrounding and we can conclude that thermal infrared overview images are potentially able to detect hot spots remotely.
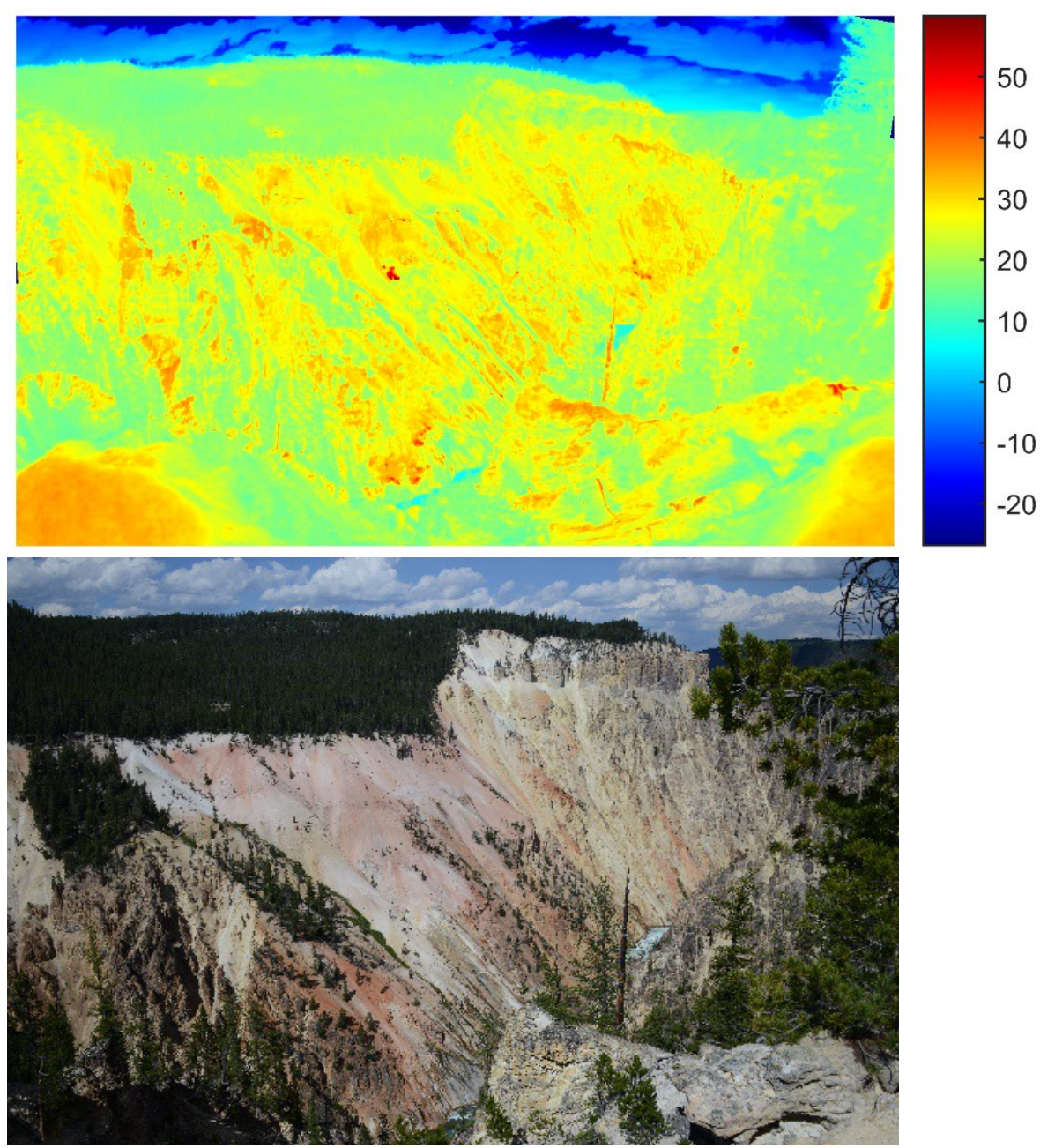

Fig. 5: Northern wall of Grand Canyon of the Yellowstone, observed from Artist point on the south rim, recorded in June 2016.

Turning around at Artist point and looking towards the West, one can see the Lower Falls of the Yellowstone River at a distance of around $1400 \mathrm{~m}$. This view (Fig. 6, VIS image) is also one of the most spectacular ones and one of the most frequently photographed scenes in the park. In addition to the VIS photo, Fig. 6 shows a long wave thermal Infrared image (top) and a NIR photo (bottom right).

The LWIR images of Fig. 6 were collected with a FLIR Tau2 336 LWIR camera with a $17^{\circ}$ field-of-view lens. It is a composite of 11 partially overlapping images, each containing $324 \times 256$ pixels with the cropped composite containing about $690 \times 482$ pixels. The spatial resolution within the thermal IR image is of course rather poor (for the $17^{\circ} \mathrm{FOV}$ is around $0.91 \mathrm{mrad}$ per pixel). Still, the IR image nicely reveals the colder parts with the river and its cold water spray of around $15^{\circ} \mathrm{C}$. In addition, it reveals a very prominent dark blue spot with a temperature of approximately $0{ }^{\circ} \mathrm{C}$ at the left lower side of the falls. In the VIS image this area was of white color and could not be differentiated from the water and its spray. In the IR it is obvious that this must be a patch of snow or ice that survived melting during the springtime. 


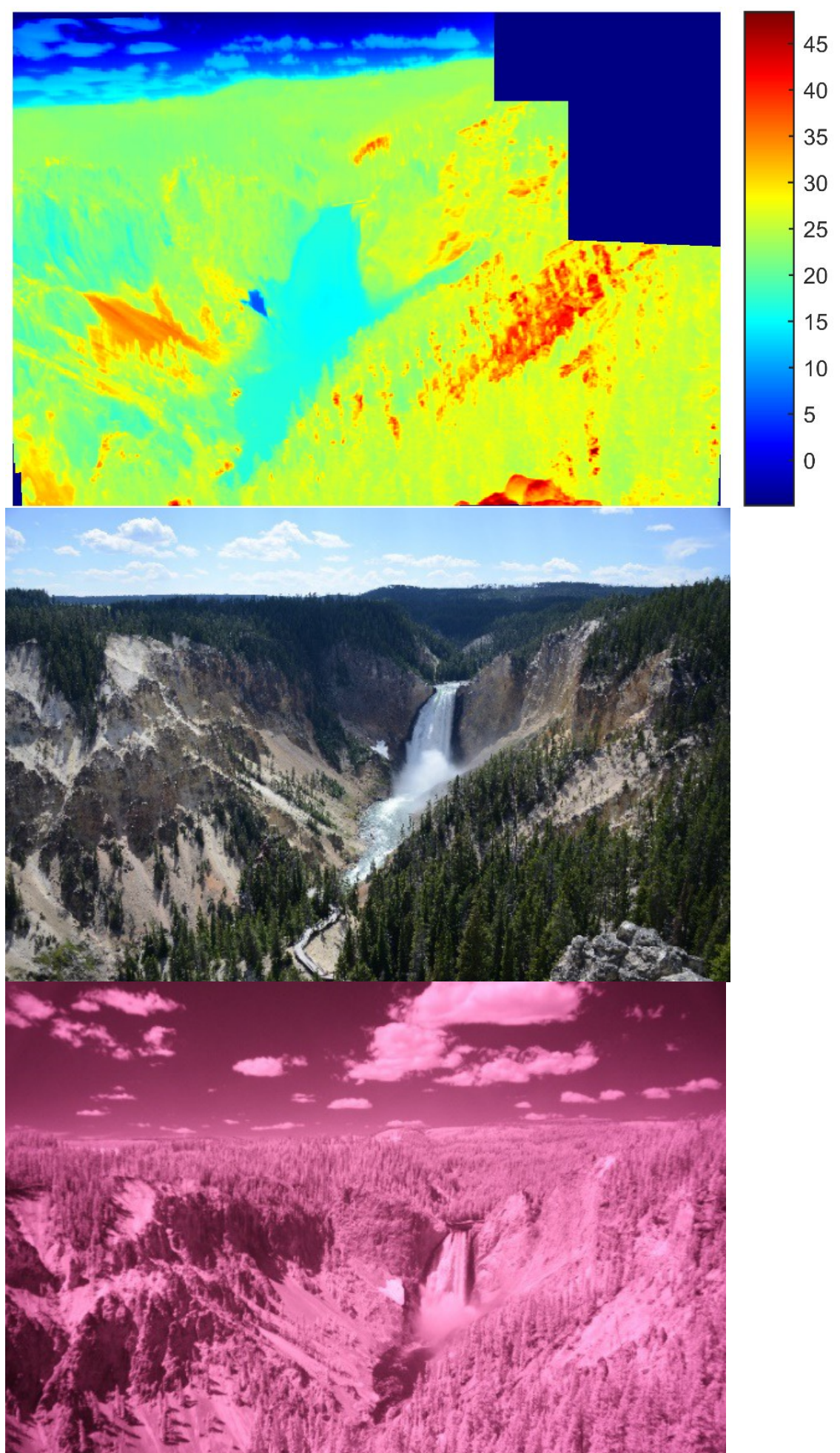

Fig. 6: Lower Falls of the Yellowstone river as observed from Artist point on the south rim. As seen in thermal Infrared (top) visible light (bottom left) and NIR radiation (bottom right). 
Finally, we recorded a near-infrared (NIR) image with a Canon EOS1000D DSLR camera that was modified by removing the NIR-blocking filter and adding a VIS-blocking filter. The result of this modification is that radiation is detected starting at a wavelength near $800 \mathrm{~nm}$ and extending up to $1100 \mathrm{~nm}[13,14]$. NIR cameras mostly detect the available scattered radiation, similar to VIS cameras. At NIR wavelengths, thermal radiation for typical near-ambient temperatures of rivers, rocks, and even hot pools of water is so small that it is buried below the scattered sunlight. In examining the NIR image, we first notice that the NIR camera image shows very pronounced contrast between sky and clouds $[13,14]$. Second, water and ice scatter NIR radiation differently, which is why the ice sheet is also very clearly visible in the NIR image.

\section{Differences of optical features between VIS and IR images}

There are quite a few differences in observable objects when comparing IR and VIS images. One particularly striking example in nature is enlarged in Fig. 7. In the vicinity of Grand Prismatic Spring in the Midway Geyser Basin, large areas around the spring were flooded with the outflow water. In the past, before being flooded, there were plants and trees. Then, many years ago, the specific area of Fig. 7, being several hundred meters away from the spring, was flooded. The hot water cooled down rapidly, but it contaminated the soil with Sulphur. As a result, all plants and trees died. Now we see only dead lodgepole pine tree trunks, which stick out of the soil. They provide fascinating optical phenomena.

Let us carefully compare the IR and visible images in Fig. 7. There are similarities, but also differences between the two. The smooth water surface provides a good mirror and both images are similar in containing reflections of the trees on the smooth water surface, pointing at the camera from the base of each tree. In the visible, the trunks are darker than the soil and so are the reflections. However, in the thermal infrared the tree trunks appear brighter, as do the reflections. The reason is simple: it was a clear and sunny day and the trees were heated by solar radiation for several hours before we recorded this image. Therefore, the trunks were much warmer than the water-covered soil, which had a temperature near $30{ }^{\circ} \mathrm{C}$.

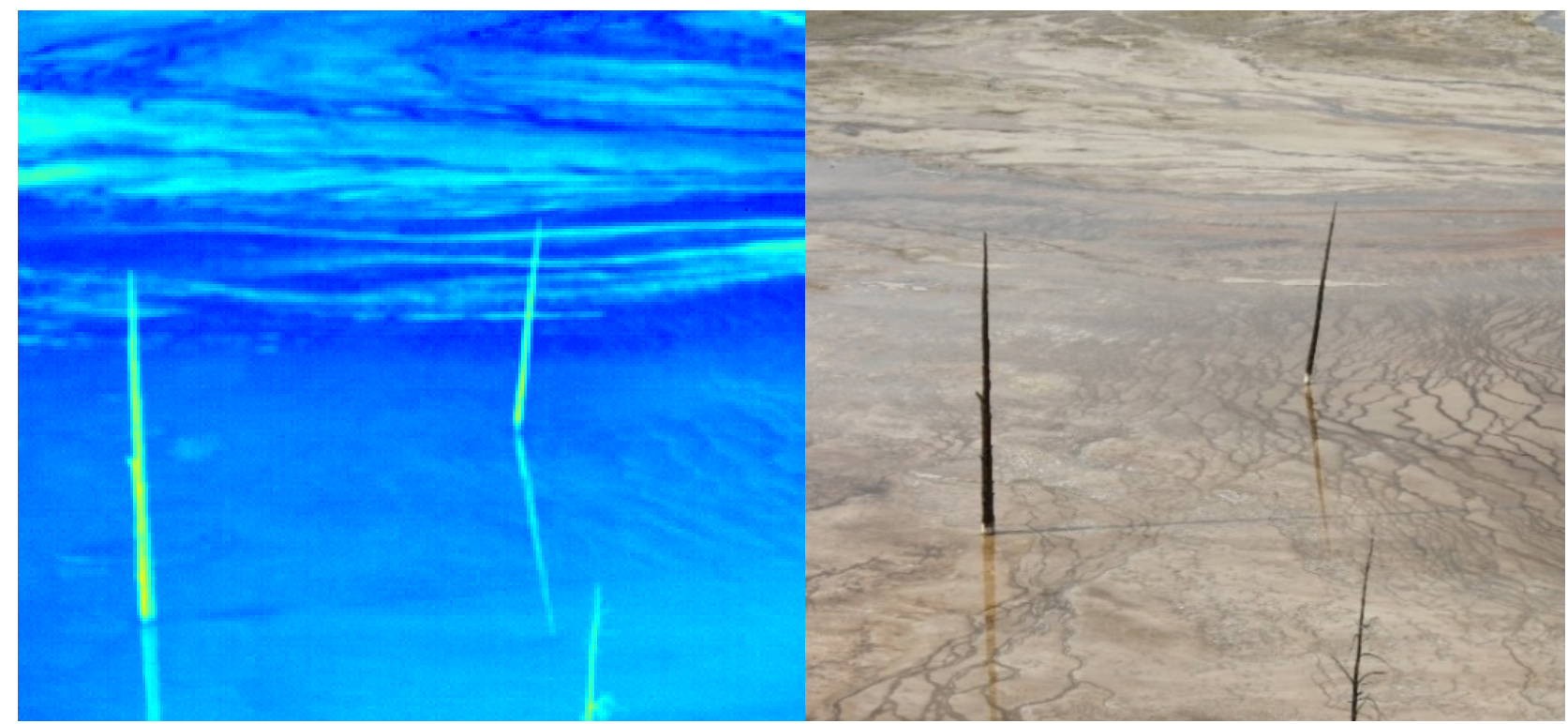

Fig. 7: Dead lodgepole pine tree trunks in the Midway Geyser Basin near Grand Prismatic Spring create specifically differing optical features when comparing the IR (left) with the respective visible scene (right).

Now, look carefully to see that there is also something else very different! The two tree trunks in the visible image also are casting shadows on the water, pointing away from the sun, which is to the left. These shadows extend to the right, at an angle near $90^{\circ}$ with respect to the tree reflections. These very prominent shadows in the visible images are more or less missing in the infrared scene. The reason is again due to the underlying differing physics. In the IR the primary source of radiation is thermal emission, not sunlight. Furthermore, in surfaces with high thermal conduction, like water 
and wet soil, the time-varying contribution of the ground heating or cooling due to the moving shadows is small compared to the overall ground emission. Therefore, the IR shadow is hardly observable, if at all.

Another final example where the images recorded in different spectral regions show pronounced differences is shown in Fig. 8. It depicts Morning Glory Pool, a very prominent silent pool in the park, which is particularly famous for its colorful yellow-orange-green appearance [6,7]. The left image is again a near infrared image, recorded with a Canon EOS1000D camera modified by removing the NIR-blocking filter and adding a VIS-blocking filter. As also seen previously, NIR cameras are not sensitive enough to detect thermal radiation from the hot pools, even at their quite elevated temperatures (compared to ambient).

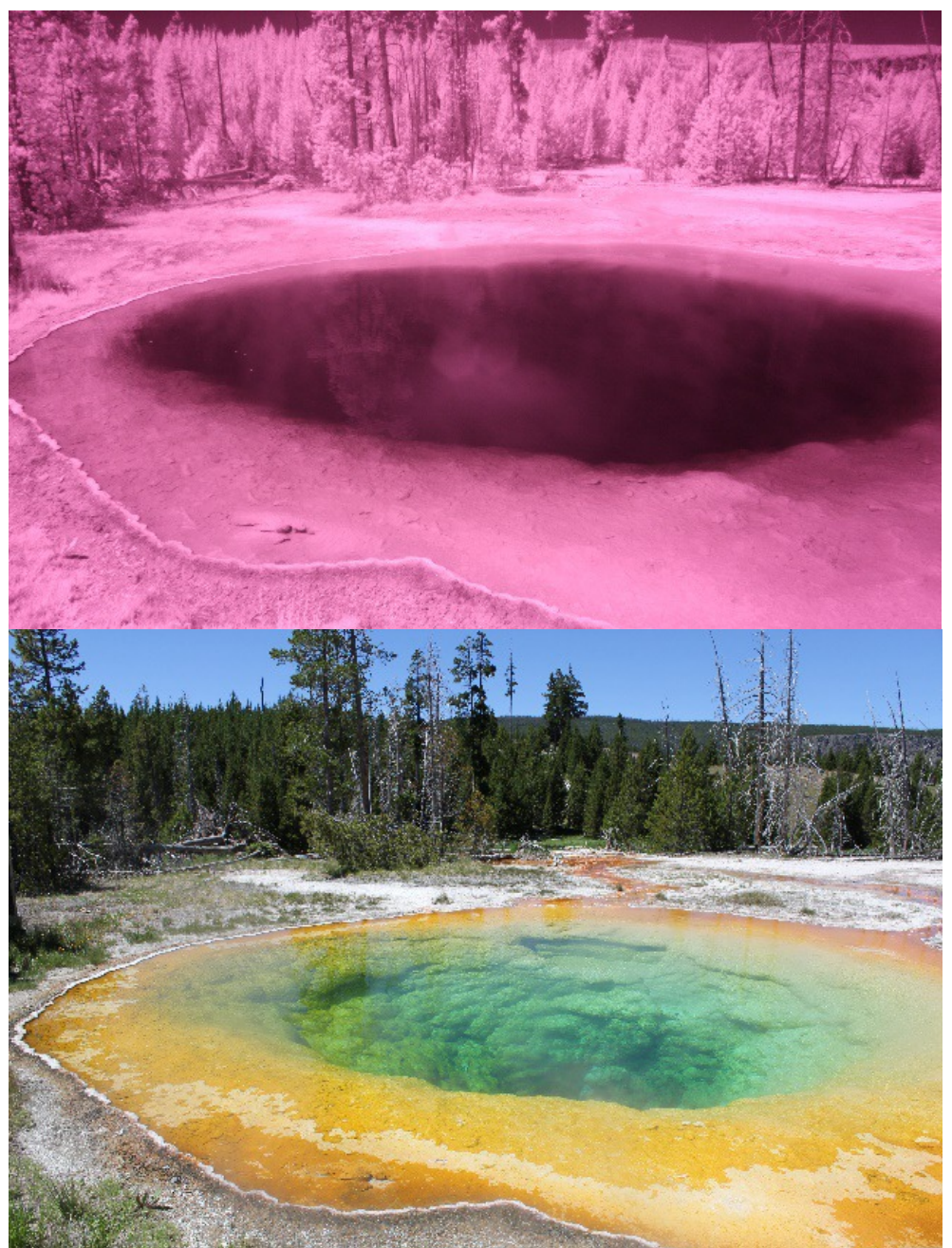

Fig. 8: Morning Glory Pool in NIR and VIS spectral range.

There are very pronounced differences between the two images. The dark green vegetation in the visible appears very bright and white in the NIR photo. As a consequence, the pool also appears different. In the VIS, one readily sees a variety of pool colors, which are related to both water temperature and water depth [6,7]. However, the NIR image is more or less monochrome and does not differentiate between the observed differing colors in the VIS. The pool surface appears very dark with one exception: in the center there are some whitish reflections and a closer look reveals that one is able to detect reflection images of the bright trees and probably also some white clouds that are not even in the field of view. In the VIS images, these reflections are not seen for two reasons. First, the vegetation which provides the scattered light source is dark and second, the background within the pool consist of bright rocky walls with a lot of light scattering. Therefore, the small reflection contribution of trees or clouds cannot be seen in the VIS image. 


\section{CONCLUSION}

Thermal IR cameras open our eyes to a new, temperature-dependent version of the world we are accustomed to seeing with visible light. Yellowstone National Park serves as a great laboratory, rich with thermal features to observe with IR imagery. Some similarities exist, such as the general shapes and sizes of objects and their reflections, but many differences also exist. Examples shown here included the ability to measure the temperature of hot water, the variation of emissivity with viewing angle, and discovery of cold snow hidden in the shadows and hot gases leaking from seemingly solid canyon walls. The educational and outreach opportunities are many with such images.

\section{Acknowledgments:}

Funding for these explorations was provided by the Montana State University Optical Technology Center.

Several of the student authors were participants in a summer Research Experience for Undergraduates (REU) program at Montana State University, funded by the National Science Foundation, grant \#1156011.

\section{REFERENCES}

[1] M. Vollmer and K.-P. Möllmann, [Infrared Thermal Imaging: Fundamentals, Research and Applications], Wiley-VCH, Weinheim, (2010), $2^{\text {nd }}$ extended and revised edition to be published in 2017.

[2] M. Vollmer and K.-P. Möllmann, "Teaching physics and understanding infrared thermal imaging," Education and Training in Optics and Photonics (ETOP) 2017, Proc. of SPIE, this issue

[3] J. A. Shaw and P. W. Nugent, "Physics principles in radiometric infrared imaging of clouds in the atmosphere," Eur. J. Phys. 34 S111-S121 (2013).

[4] J. A. Shaw, P. W. Nugent, and M. Vollmer, "Infrared moon imaging for remote sensing of atmospheric smoke layers," Applied Optics 54(4), B64-B75 (2015).

[5] M. Vollmer, J. A. Shaw, and P. W. Nugent, "Visible and invisible mirages: Comparing inferior mirages in the visible and thermal infrared", Applied Optics 54(4), B76-B84 (2015).

[6] P. W. Nugent, J. A. Shaw, and M. Vollmer, "Colors of thermal pools at Yellowstone National Park," Appl. Opt. 54(4), B128-B139 (2015).

[7] J. A. Shaw, P. W. Nugent, and M. Vollmer, "Colors of the Yellowstone Thermal Pools for Teaching Optics," Education and Training in Optics and Photonics (ETOP) 2015, edited by Eric Cormier, Laurent Sarger, Proc. of SPIE Vol. 9793, 97931S (2015).

[8] J. A. Shaw, P. W. Nugent, W. Harris, and M. Vollmer, "Infrared Yellowstone", Opt. Photon. News 28(6), 36-43 (2017).

[9] J. A. Shaw, Optics in The Air: Observing Optical Phenomena Through Airplane Windows, SPIE Press (2017).

[10] P. W. Nugent, J. A. Shaw, and N. J. Pust, "Correcting for focal-plane-array temperature dependence in microbolometer infrared cameras lacking thermal stabilization,” Opt. Eng. 52(6), 061304 (2013).

[11] P. W. Nugent, and J. A. Shaw, "Calibration of uncooled LWIR microbolometer imagers to enable long-term field deployment," Proc. SPIE 9071, 90710V (2014).

[12] Some IR video files are available as supplementary material on the Optics and Photonics News (OPN) website: https://www.osa-opn.org/home/

[13] K. Mangold, J. A. Shaw, and M. Vollmer, “The Physics of Near-Infrared Photography,” Eur. J. Phys. 34(6), S51-71 (2013).

[14] M. Vollmer, K.-P. Möllmann, and J. A. Shaw, "The optics and physics of near infrared imaging," in Education and Training in Optics and Photonics (ETOP) 2015, edited by Eric Cormier, Laurent Sarger, Proc. SPIE 9793, 97930Z (2015). 